\title{
Prostate-specific antigen screening can be beneficial to younger and at-risk men
}

\author{
Monique J. Roobol PhD Msc, Chris H. Bangma MD PhD, Stacy Loeb MD
}

I n 2008, the United States Preventive Services Task Force issued guidelines recommending against screening for prostate-specific antigen (PSA) in men aged 75 years or older. They concluded that there was insufficient evidence as to the benefits and harms of screening in younger men. The task force recently performed a literature review and, in October 2011, issued a new draft recommendation against PSA screening (grade D, fair evidence against) for men of all ages (www.uspreventiveservicestaskforce.org /announcedetails.htm). ${ }^{1,2}$

Although the final version is not yet published, the draft differs from recommendations published by many other professional organizations. The American Cancer Society recommends that men make an informed decision with their health care provider about whether they should be screened for prostate cancer. ${ }^{3}$ The American Urological Association similarly recommends that men with a life expectancy of at least $10-15$ years should be given a choice about screening. ${ }^{4}$ The National Comprehensive Cancer Network in the US recommends discussing the risks and benefits before offering screening. ${ }^{5}$ In the United Kingdom, there is no organized screening program for prostate cancer; instead, an informed-choice program, Prostate Cancer Risk Management, has been introduced. ${ }^{6}$ The Cancer Council Australia decided that the benefit of prostate cancer screening is yet unproven and does not support population-based screening for men who have no symptoms. The Council recommends a patient-centred approach for individual decisions about PSA testing.?

In Canada, the Canadian Task Force on Preventive Health Care, the counterpart to the US Preventive Services Task Force, last reported on PSA screening in 1994. At that time, they recommended against PSA screening (grade D) ${ }^{8} \mathrm{~A}$ new draft recommendation from the Canadian task force is expected in 2013. Meanwhile, the Genitourinary Tumour Group of the British Columbia Cancer Agency and the Vancouver Prostate Centre recommend PSA testing for men with no symptoms who are well-informed about the risks of overdiagnosis and overtreatment, but who still wish to pursue the benefits of early diagnosis. ${ }^{9}$ In addition, the Canadian Urological Association recently published recommendations for screening stating that the harms and benefits of PSA testing should be explained to all patients. ${ }^{10}$ One of the key differences between these guidelines and the draft statement of the US Preventive Services Task Force is that a grade D recommendation against screening does not promote a patient-centred decision-making process. A grade $\mathrm{C}$ recommendation, however, allows for patient participation in the decision through a discussion of the risks and benefits, which is in line with the available evidence. ${ }^{11}$

\section{Results of recent research}

The US Preventive Services Task Force should be commended for its comprehensive literature review. To assess the new draft recommendation, it is critical to examine the recent evidence on PSA screening. Since the 2008 version was issued, the results of several randomized trials have become available.

The largest of these studies is the European Randomized Study of Screening for Prostate Cancer, which included 162243 men aged 5569 years, and provided high-quality evidence that screening reduces prostate cancer-specific mortality. ${ }^{12}$

Men randomized to the screening arm of the study were screened every four years (every two years in the Swedish centre) and biopsy was usu-

\section{- Ker pOINTS}

- The United States Preventive Services Task Force recently issued a draft recommendation against routine screening for prostate cancer.

- Level 1 evidence from the largest randomized trial of screening showed that testing for prostate-specific antigen reduces the rates of advanced disease and prostate cancer-specific mortality.

- Shared decision-making is an important component of screening, which is incorporated into many other professional guidelines.

- In addition to the total level of prostate-specific antigen, clinical decisions regarding the need for diagnostic work-up should be guided by patient-specific factors including age, race, family history and prostate volume. 
ally recommended for a PSA level of $3.0 \mathrm{ng} / \mathrm{mL}$ or higher. After a median follow-up of nine years, the study showed a significant reduction in prostate cancer mortality of $20 \%$ (rate ratio [RR] $0.80,95 \%$ confidence interval $[\mathrm{CI}] 0.65-0.98)$ in favour of screening, ${ }^{12}$ and a $31 \%$ reduction (RR $0.69,95 \%$ CI $0.51-0.92$ ) after correction for noncompliance and contamination. ${ }^{13}$ To prevent one death from prostate cancer, the number needed to invite was 1410 , and the number needed to detect was 48 . A new update with a median follow-up of 11 years confirmed the reduction in prostate cancer mortality with screening (RR 0.79, 95\% CI 0.68-0.91). ${ }^{14}$ To prevent one death from prostate cancer, the number needed to invite was reduced to 936 , and the number needed to detect dropped to $33 . .^{14}$

After its publication, this screening study was criticized for having treatment differences between its two arms, making it difficult to distinguish the effect of screening on prostate cancerspecific mortality. Interestingly, after correcting for potential confounders like age, PSA level, and tumour stage and grade at the time of diagnosis, there was actually a small treatment advantage for men with high-risk prostate cancer who had been randomized to the control arm of the study; however, treatment differences were shown to play a smaller role in outcome than tumour features. ${ }^{15}$

Updated results were recently published from the Prostate, Lung, Colorectal and Ovarian Cancer Screening Trial. ${ }^{16}$ The prostate arm of this study is a screening trial in which men aged 5574 years $(n=76693$ from 10 centres in the US) were randomly assigned to receive either annual screening (38 343 men) or usual care (i.e., the control arm; 38350 men). Screening consisted of annual PSA testing for six years and digital rectal examination for four years. The type of follow-up evaluation (i.e., biopsy of the prostate) was not mandated by the protocol and was determined in conjunction with individual health care providers. Results were reported after a median of seven years' follow-up and showed no reduction in prostate cancer-specific mortality with screening (RR 1.13, 95\% CI 0.75-1.70). ${ }^{17}$ These findings were recently confirmed with $92 \%$ of participants followed to 10 years. ${ }^{16}$

Compared with the European Randomized Study of Screening for Prostate Cancer, several factors in the Prostate, Lung, Colorectal and Ovarian Cancer Screening Trial may help to explain the divergent results, including high rates of PSA screening before entry to the study, frequent screening in the control arm during the study and poor compliance with biopsy recommendations. However, it should be noted that prostate cancerspecific mortality in the Prostate, Lung, Colorectal and Ovarian Cancer Screening Trial at 10 years was $25 \%$ lower among patients who had undergone PSA testing twice or more at baseline than among those who had not been tested previously. ${ }^{18,19}$

In addition to these contemporary trials, the literature review done by the US Preventive Services Task Force cited a meta-analysis by Djulbegovic and colleagues..$^{20}$ These authors found that screening was associated with an increased probability of receiving a diagnosis of prostate cancer (RR 1.46, 95\% CI 1.21-1.77) and stage I prostate cancer (RR 1.95, 95\% CI 1.22-3.13). There was no significant effect of screening on death from prostate cancer (RR $0.88,0.71-1.09$ ) or overall mortality (RR 0.99 , 0.97-1.01). However, a substantial limitation of this meta-analysis is the attempt to combine data from the European Randomized Study of Screening for Prostate Cancer and the Prostate, Lung, Colorectal and Ovarian Cancer Screening Trial despite their differences. In addition, the meta-analysis includes older trials that do not meet sufficient quality criteria. ${ }^{21}$ One of these trials, from Norrkoping, Sweden, involved screening every three years exclusively by digital rectal examination, with PSA testing eventually added during the third round of screening. ${ }^{22}$ In addition, the meta-analysis by Djulbegovic and colleagues included data from the Quebec randomized trial, ${ }^{23}$ in which only $23 \%$ of men randomized to the intervention arm received screening, and the analysis was not done in an intent-to-treat fashion. The drawbacks of these studies and their different screening protocols limit their utility to inform modern screening guidelines..$^{21,24}$

\section{Interpreting the results of screening trials}

\section{Mortality}

The media response to the draft recommendation of the US Preventive Services Task Force has largely focused on how PSA screening has not been shown to reduce overall mortality. ${ }^{25} \mathrm{Be}$ cause PSA screening should be limited to men with a life expectancy of at least 10 years, the difference in mortality during the first 10 years is less relevant to screening decisions. Moreover, cancer-specific mortality, not overall mortality, is the primary outcome in screening trials. Because deaths from prostate cancer are a small proportion of all deaths, comparisons of overall mortality are underpowered. Thus, a screening program that reduces cancer-specific mortality should not be stopped because of a lack of reduction in overall mortality. ${ }^{26}$

The primary outcome of the European Ran- 
domized Study of Screening for Prostate Cancer was prostate cancer-specific mortality, which was $20 \%$ lower with screening at nine years' follow-up in the intent-to-treat analysis. ${ }^{12}$ In the original publication, with a median of nine years' follow-up, the survival curves had just begun to diverge between the screening and control groups $;{ }^{12}$ the number needed to invite and diagnose have further decreased with time. ${ }^{13}$ In a randomized screening trial from Goteborg, ${ }^{27}$ the $\mathrm{RR}$ for prostate cancer death in favour of screening was 0.56 (95\% CI $0.39-0.82)$ at 14 years, and only 12 men had to be screened and given a diagnosis to prevent 1 death from prostate cancer. Owing to the long natural history of screendetected prostate cancer, additional follow-up from screening trials will provide a more useful assessment of the benefits associated with PSA testing in terms of reduced mortality.

\section{Metastatic disease}

Mortality is not the only important outcome of a screening trial. In the European Randomized Study of Screening for Prostate Cancer, screening resulted in a $41 \%$ reduction in metastatic disease at diagnosis $(p<0.001) .{ }^{12}$ In a comparison between the European Randomized Study of Screening for Prostate Cancer and a population from Northern Ireland, where screening is rare, van Leeuwen and coauthors showed that the relative risk of metastasis in the European population was 0.47 (95\% CI $0.35-0.63, p<0.001)$ compared with the population from Northern Ireland. ${ }^{28}$

The Goteborg trial similarly showed that screening reduced metastatic disease (46 men in the screening group v. 87 men in the control group; $p=0.0003$ ) and the number of men requiring endocrine treatment for prostate cancer. ${ }^{27}$ The latter issue is important, because the Randomized Scandinavian Prostate Cancer Group Study Number 4 showed a significant negative impact on mood, sense of well-being and self-assessed quality of life with androgendeprivation therapy for progressive disease. ${ }^{29}$ Overall, metastatic disease has substantial adverse effects on quality of life, and there is a paucity of durable treatment options for advanced prostate cancer. Thus, preventing metastatic disease should be recognized as an important benefit of screening.

\section{A possible way forward: individualized decision-making}

Screening is not for everyone. For this reason, the individual decision-making process is important. For elderly men with multiple medical comorbidities, the harms of screening may outweigh the benefits. Yet, it still occurs. For example, Walter and coauthors report that among men aged 85 years and older in poor health who were seen at 104 US Department of Veterans Affairs facilities in 2002-03, 36\% underwent PSA testing. ${ }^{30}$ These men had limited life expectancy and were unlikely to benefit from early detection and treatment. ${ }^{30}$ Moreover, older men with multiple comorbidities have a greater risk of complications from both the diagnostic work-up (biopsy) ${ }^{31}$ and treatment. ${ }^{32}$

In contrast, the risk-to-benefit ratio shifts dramatically for healthy men with a long life expectancy. In the Prostate, Lung, Colorectal and Ovarian Cancer Screening Trial, older age at randomization was associated with increased risk of dying from prostate cancer, even in the screening arm. ${ }^{17}$ Men in the screening arm aged 55-64 years at the time of randomization had a rate of death from prostate cancer of 2.35 per 10000 person-years, whereas the rate for men aged 6574 years at the time of randomization was 6.17 per 10000 person-years. ${ }^{17}$ Furthermore, despite no survival advantage with screening in the overall population, ${ }^{17}$ a follow-up analysis found that screening significantly reduced disease-specific mortality in men with minimal comorbidity (adjusted hazard ratio [HR] 0.56, 95\% CI $0.33-$ 0.95). ${ }^{33}$ The authors reported that only five healthy men needed to be screened and given treatment to prevent one death from prostate cancer. In the updated analysis, the authors used a more limited definition of comorbidity, and found no difference in survival outcomes (relative risk $1.00,95 \%$ CI $0.76-1.31$ ); however, using their original criteria, there remained a statistically significant $27 \%$ relative reduction in prostate cancer mortality for men with no comorbidity ${ }^{16}$ Unfortunately, the study was not powered to address this question, and these post hoc analyses must be interpreted with caution.

There is also evidence that younger men have the most to gain from treatment. One randomized trial showed that radical prostatectomy was associated with a significant improvement in overall survival (relative risk $0.52,95 \%$ CI 0.37 0.73), prostate cancer survival (relative risk 0.49 , 95\% CI 0.31-0.79) and metastasis-free survival (relative risk $0.47,95 \%$ CI 0.32-0.70) compared with watchful waiting in men less than 65 years of age..$^{34}$ It is noteworthy that the survival advantages associated with definitive therapy persisted in the subset of patients with low-risk disease. Results from the Prostate Cancer Intervention versus Observation Trial ${ }^{35}$ are forthcoming and will also shed light on this issue.

With respect to the risks of screening, young 
healthy men are at lower risk for complications as a result of a biopsy ${ }^{31}$ and treatment-related adverse effects ${ }^{32}$ (such as erectile dysfunction and incontinence) than older men.

These combined results show that individual factors such as age and comorbidity have a substantial impact on the balance of the benefits and harms of screening. Overuse of screening for unhealthy men with a limited life expectancy should indeed be discouraged; however, the same does not hold true for young healthy men. As quoted from a statement by the American Urological Association:

[J]ust as it is inappropriate to issue a "one size fits all" pro-screening message, it is equally inappropriate, and potentially irresponsible, to issue a blanket statement against testing, as studies have demonstrated strong benefits to prostate cancer screening. We believe that there is strong evidence that, for some men - generally those younger and in good health — testing saves lives. ${ }^{4}$

Considering individual factors in the screening decision is important for several reasons. For example, the US task force's draft recommendation applies to all men, regardless of risk factors such as race. Black men have a greater risk of receiving a diagnosis of prostate cancer and higher disease-related mortality; however, they represented only $5 \%$ of the population involved in the Prostate, Lung, Colorectal and Ovarian Cancer Screening Trial, and were not included in several other randomized screening trials. ${ }^{12,17,27}$ Thus, the generalizability of these studies to black men may be limited. If the benefits of screening are greater for high-risk populations, categorically discouraging screening has the potential to increase disparities in health care.

Multiple factors influence the risk of prostate cancer (and aggressive disease) in a given patient in addition to the level of PSA. These factors include age, race, the results of a digital rectal examination, family history and prostate volume. As a result, multivariable predictive tools are increasingly used in clinical practice to guide the decisions for screening and biopsy. ${ }^{36,37}$ An example is the "risk calculator" developed by the European Randomized Study of Screening for Prostate Cancer (available at www.prostatecancer -riskcalculator.com or www.uroweb.org). The "calculator" is a set of seven different calculators, ranging from one suitable for lay people (requiring basic information such as age and family history) to one that uses pathologic data to predict the risk of potentially indolent prostate cancer. The risk calculators that predict the outcome of a biopsy (i.e., having prostate cancer or potentially aggressive prostate cancer) have been externally validated in several populations and successfully implemented in clinical practice. ${ }^{38,39}$

Considering multiple risk factors in combination can provide patients with a more personalized risk assessment and reduce the number of unnecessary biopsies versus using PSA testing alone. However, as PSA is an important component in each of these models, eliminating PSA screening would preclude their use.

Finally, the literature review performed by the US Task Force extensively described the risks of treatment in its review on the potential future harms associated with screening. ${ }^{1}$ However, not all screen-detected prostate cancer requires treatment. ${ }^{40}$ Indeed, the Goteborg trial showed a $44 \%$ reduction in prostate cancer-specific mortality (RR $0.56,95 \%$ CI $0.39-0.82$ ), despite $30 \%$ of cancers detected during screening being on active surveillance (rather than treatment) at the last follow-up. ${ }^{27}$ Although $60 \%$ of men from the Goteborg trial were included in the report from the European Randomized Study of Screening for Prostate Cancer, the Goteborg trial is different in several important ways from the other sites, including a younger population, screening every two years and longer follow-up, which might account for the improved outcomes.

Many prognostic tools are commonly used to assist patients and physicians with deciding whether or not to pursue treatment. ${ }^{41-43}$ PSA levels, histology and staging results are used to stratify the risk of progressive disease and guide decisions. In the absence of screening, many cancers would be detected too late for patients to benefit from the full spectrum of treatment or conservative strategies, corresponding with a resurgence of metastatic disease.

\section{Conclusion}

Rather than abandoning a screening test that reduces death and suffering, efforts should be focused on selecting patients more carefully. Screening should be encouraged for healthy younger men and men with risk factors (e.g., black ancestry, positive family history) and discontinued for elderly men with multiple comorbidities and limited life expectancy. In addition, improvements in diagnostic testing and treatment can reduce the potential associated harms for men with a sufficient life expectancy who choose to undergo screening.

Greater benefits of screening will likely emerge with the additional follow-up from the ongoing randomized trials; meanwhile, investigation into more specific biomarkers, the use of multivariable predictive tools in decisionmaking, increased use of conservative management for low-risk patients and ongoing techno- 
logical advances in treatment can help to further improve the ratio of benefits to harms.

\section{References}

1. Chou R, Croswell JM, Dana T, et al., Screening for prostate cancer: a review of the evidence for the US Preventive Services Task Force. Ann Intern Med 2011;155:762-71.

2. US Preventive Services Task Force. Screening for prostate cancer: draft recommendation statement. Rockville (MD): US Preventive Services Task Force; 2011. Available: www.uspreventiveservices taskforce.org/uspstf12/prostate/draftrecprostate.htm (accessed 2011 Oct. 29).

3. American Cancer Society. American Cancer Society recommendations for prostate cancer early detection. Atlanta (GA): The Society; 2012. Available: www.cancer.org/Cancer/ProstateCancer /MoreInformation/ProstateCancerEarlyDetection/prostate-cancer -early-detection-acs-recommendations (accessed 2011 May 21).

4. American Urological Association. AUA response to 2011 US Preventive Services Task Force draft recommendations on prostate cancer testing. Linthicum (MD): The Association; 2011. Available: www.auanet.org/content/health-policy/government -relations-and-advocacy/in-the-news/aua-response-to-uspstf.cfm ?acid=7333870728|NE21Off717\&WT.mc_id=NetNews7006. (accessed 2011 Nov. 9).

5. National Comprehensive Cancer Network clinical practice guidelines in oncology. Available: www.nccn.org/professionals /physician_gls/f_guidelines.asp. Fort Washington (PA): National Comprehensive Cancer Network; 2011. (accessed 2011 June 25).

6. Prostate Cancer Risk Management Programme. Sheffield (UK) 2012. Available: www.cancerscreening.nhs.uk/prostate/ (accessed 2012 Feb. 3)

7. Cancer Council Australia. Position statement: prostate cancer screening. Surry Hills (NSW): The Council; 2008. Available: www.cancer.org.au/File/PolicyPublications/Position_statements /PS-Prostate cancer screening Apr08.pdf. (accessed 2012 Feb. 3).

8. Feightner JW. Screening for prostate cancer. London $(\mathrm{ON})$ : The Canadian Task Force on Preventive Health Care; 1994.

9. BC Cancer Agency. PSA screening. Vancouver (BC): The Agency; 2010. Available: www.bccancer.bc.ca/HPI/Cancer ManagementGuidelines/Genitourinary/Prostate/PSAScreening /default.htm (accessed 2011 Nov. 11).

10. Izawa JI, Klotz L. Siemens DR, et al. Prostate cancer screening. Canadian guidelines 2011. The Canadian Urological Association; 2011. p. 1-26. Available: www.cua.org/userfiles/files/CUA $\% 20 \mathrm{PCa} \% 20$ Screening\%20Guidelines\%20v3(4).pdf (accessed 2012 Mar. 7).

11. McNaughton-Collins MF, Barry MJ. One man at a time resolving the PSA controversy. N Engl J Med 2011:365:1951-3

12. Schröder FH, Hugosson J, Roobol MJ, et al. Screening and prostate-cancer mortality in a randomized European study. $N$ Engl J Med 2009:360:1320-8

13. Roobol MJ, Kerkhof M, Schröder FH, et al. Prostate cancer mortality reduction by prostate-specific antigen-based screening adjusted for nonattendance and contamination in the European Randomized Study of Screening for Prostate Cancer (ERSPC). Eur Urol 2009;56:584-91.

14. Schröder FH, Hugosson J, Roobol MJ, et al. Prostate-cancer mortality at 11 years of follow-up. N Engl J Med 2012;366:981-90.

15. Wolters T, Roobol MJ, Steyerberg EW, et al. The effect of study arm on prostate cancer treatment in the large screening trial ERSPC. Int J Cancer 2010;126:2387-93.

16. Andriole GL, Crawford ED, Grubb RL 3rd, et al. Prostate cancer screening in the Randomized Prostate, Lung, Colorectal, and Ovarian Cancer Screening Trial: mortality results after 13 sears of follow-up. J Natl Cancer Inst 2012;104:125-32.

17. Andriole GL, Crawford ED, Grubb RL 3rd, et al. Mortality results from a randomized prostate-cancer screening trial. $N$ Engl J Med 2009;360:1310-9.

18. Cooperberg MR, Carroll PR. Prostate-cancer screening. $N$ Engl J Med 2009;361:203, author reply 204-5.

19. Catalona WJ. Prostate-cancer screening. N Engl J Med 2009; 361:202; author reply 204-5.

20. Djulbegovic M, Beyth RJ, Neuberger MM, et al. Screening for prostate cancer: systematic review and meta-analysis of randomised controlled trials. BMJ 2010;341:c4543.

21. Roobol MJ, Carlsson S, Hugosson J. Meta-analysis finds screening for prostate cancer with PSA does not reduce prostate cancerrelated or all-cause mortality but results likely due to heterogeneity - the two highest quality studies identified do find prostate cancer-related mortality reductions. Evid Based Med 2011;16:20-1.

22. Sandblom G, Varenhorst E, Rosell J, et al. Randomised prostate cancer screening trial: 20 year follow-up. BMJ 2011;342:d1539.
23. Labrie F, Candas B, Cusan L, et al. Screening decreases prostate cancer mortality: 11-year follow-up of the 1988 Quebec prospective randomized controlled trial. Prostate 2004;59:311-8.

24. Loeb S, Trock BJ. Re: Randomised Prostate Cancer Screening Trial: 20 year follow-up. Eur Urol 2011;60:1306-7.

25. Harris G. US panel says no to prostate screening for healthy men. The New York Times 2011 Oct. 6. Available: www.nytimes.com /2011/10/07/health/07prostate.html. (accessed 2011 Nov. 1).

26. Steele RJ, Brewster DH. Should we use total mortality rather than cancer specific mortality to judge cancer screening programmes? No. BMJ 2011;343:d6397.

27. Hugosson J, Carlsson S, Aus G, et al. Mortality results from the Goteborg randomised population-based prostate-cancer screening trial. Lancet Oncol 2010;11:725-32.

28. van Leeuwen PJ, Connolly D, Gavin A, et al. Prostate cancer mortality in screen and clinically detected prostate cancer: estimating the screening benefit. Eur J Cancer 2010;46:377-83.

29. Johansson E, Bill-Alxelson A, Holmberg L, et al., Time, symptom burden, androgen deprivation, and self-assessed quality of life after radical prostatectomy or watchful waiting: the Randomized Scandinavian Prostate Cancer Group Study Number 4 (SPCG-4) clinical trial. Eur Urol 2009;55:422-30.

30. Walter LC, Bertenthal D, Lindquist K, et al. PSA screening among elderly men with limited life expectancies. JAMA 2006; 296:2336-42.

31. Loeb S, Carter HB, Berndt SI, et al. Complications after prostate biopsy: data from SEER-Medicare. J Urol 2011;186:1830-4.

32. Loeb S, Roehl KA, Helfand BT, et al. Complications of open radical retropubic prostatectomy in potential candidates for active monitoring. Urology 2008;72:887-91.

33. Crawford ED, Grubb R $3^{\text {rd }}$, Black A, et al. Comorbidity and mortality results from a randomized prostate cancer screening trial. J Clin Oncol 2011;29:355-61.

34. Bill-Axelson A, Holmberg L, Ruutu M, et al. Radical prostatectomy versus watchful waiting in early prostate cancer. $N$ Engl $J$ Med 2011;364:1708-17.

35. Wilt TJ, Brawer MK, Barry MJ, et al. The Prostate cancer Intervention Versus Observation Trial: VA/NCI/AHRQ Cooperative Studies Program \#407 (PIVOT): design and baseline results of a randomized controlled trial comparing radical prostatectomy to watchful waiting for men with clinically localized prostate cancer. Contemp Clin Trials 2009;30:81-7.

36. Roobol MJ, Steyerberg EW, Kranse R, et al. A risk-based strategy improves prostate-specific antigen-driven detection of prostate cancer. Eur Urol 2010;57:79-85.

37. Schröder FH. Stratifying risk - the US Preventive Services Task Force and prostate-cancer screening. N Engl J Med 2011;365: 1953-5.

38. Cavadas V, Osório L, Sabell F, et al. Prostate cancer prevention trial and European randomized study of screening for prostate cancer risk calculators: a performance comparison in a contemporary screened cohort. Eur Urol 2010;58:551-8.

39. Trottier G, Roobol MA, Lawrentschuk N, et al., Comparison of risk calculators from the Prostate Cancer Prevention Trial and the European Randomized Study of Screening for Prostate Cancer in a contemporary Canadian cohort. BJU Int 2011;108:E237-44.

40. Tosoian JJ, Trock BJ, Landis P, et al. Active surveillance program for prostate cancer: an update of the Johns Hopkins experience. J Clin Oncol 2011;29:2185-90.

41. D'Amico AV, Schultz D, Loffredo M, et al. Biochemical outcome following external beam radiation therapy with or without androgen suppression therapy for clinically localized prostate cancer. JAMA 2000;284:1280-3.

42. Partin AW, Yoo J, Carter HB, et al. The use of prostate specific antigen, clinical stage and Gleason score to predict pathological stage in men with localized prostate cancer. J Urol 1993;150: $110-4$.

43. Kattan MW, Cuzick J, Fisher G, et al. Nomogram incorporating PSA level to predict cancer-specific survival for men with clinically localized prostate cancer managed without curative intent. Cancer 2008;112:69-74.

Affiliations: From the Department of Urology (Roobol, Bangma), Erasmus University Medical Centre, Rotterdam, the Netherlands; and the Department of Urology (Loeb), New York University, New York, NY

Contributors: Monique Roobol and Stacy Loeb were responsible for the conception and design of the study, the acquisition of data and drafting the manuscript. All of the authors analyzed and interpreted the data, revised the manuscript for important intellectual content and approved the final version submitted for publication. 